\title{
Mapeamento de percepções na avaliação dos impactos do mestrado profissional no perfil do seu egresso
}

\author{
Lívia Dias de Oliveira Nepomuceno ${ }^{\mathrm{a} *}$, Helder Gomes Costa ${ }^{\mathrm{b}}$ \\ a*livia@vm.uff.br, CEFET-RJ, Brasil \\ 'hgc@vm.uff.br, UFF, Brasil
}

\begin{abstract}
Resumo
Os mestrados profissionais se constituem na modalidade mais recente de pós-graduação stricto sensu no Brasil e apresentam novas dificuldades quanto à sua avaliação. 0 presente trabalho apresenta uma abordagem baseada na avaliação de lacunas de percepção quanto ao impacto do mestrado profissional no desempenho profissional do seu egresso. A metodologia foi aplicada em uma situação específica com coleta de percepções de egressos e seus chefes na organização onde trabalham, sobre a influência de um programa de mestrado no desempenho de seus alunos. Os resultados mostraram que os participantes da pesquisa perceberam um maior impacto do curso nos aspectos que versam sobre a "autoestima" e o "perfil de pesquisador" do pós-graduado.
\end{abstract}

Palavras-chave

Avaliação. Capes. Percepção. Mestrado profissional.

\section{Introdução}

As avaliações elaboradas pelo Sistema de Avaliação dos Cursos de Pós-Graduação, implantado pela Coordenação de Aperfeiçoamento de Pessoal de Nível Superior (Capes), têm sido adotadas pelas coordenações de programas, na definição de suas estratégias de ação.

Há diversos estudos no âmbito da avaliação e classificação de desempenho dos programas de pós-graduação, classificando-os segundo classes de referência.

Duas observações distintas fornecem informações relevantes para as instituições de ensino:

- A comparação dos programas ou políticas educacionais com os critérios utilizados pelo órgão nacional responsável pela avaliação - no caso do Brasil: a Capes; e

- A captação de percepções do público interno (alunos, professores e coordenadores) e do público externo (aqueles que demandam ou são beneficiários do trabalho desenvolvido pela instituição) sobre a contribuição do curso em diversos aspectos considerados importantes no desempenho profissional do aluno.

Nesse contexto, uma problemática recente é a avaliação de cursos de pós-graduação na modalidade mestrado profissional. Conforme reportado em Brasil (1998), o mestrado profissional é a designação do curso que enfatiza estudos e técnicas diretamente voltadas ao desempenho de um alto nível de qualificação profissional, que tem por objetivo a capacitação para o mercado de trabalho. Ainda segundo a Capes (COORDENAÇÃO..., 2007), esse tipo de curso se justifica pela necessidade de formação de recursos humanos para atuação fora da universidade. 0 mestrado profissional apresenta uma situação peculiar: concede aos seus egressos os mesmos direitos e prerrogativas garantidos aos oriundos do mestrado acadêmico; no entanto, pela sua definição, apresenta algumas diferenças marcantes em relação ao perfil do seu egresso, pois propõe a formação de profissionais com foco no exercício profissional no ambiente produtivo. 
0 objetivo geral desta pesquisa é contribuir na investigação sobre os impactos originados pelos cursos de pós-graduação no desempenho profissional dos seus egressos.

Mais especificamente, este trabalho pretende:

- Identificar as percepções de discentes quanto à influência do curso no seu desempenho profissional;

- Identificar percepções provenientes do meio organizacional quanto à influência do curso no desempenho de seus profissionais; e

- Mapear e detectar as lacunas de percepção entre os dois grupos entrevistados, de forma a permitir uma análise dessas lacunas.

\section{Revisão bibliográfica}

Há diversos textos que apresentam reflexões acerca dos critérios utilizados em sistemas de avaliação da pós-graduação e, também, de instituições de ensino superior (IES). A seguir comentam-se alguns desses textos, focando a avaliação de programas de mestrado profissional e a aplicação de técnicas de análise estatística multivariada, análise envoltória de dados e análise multicritério.

- Piquet, Leal e Terra (2005) discutem sobre a contribuição do mestrado profissional na qualidade da formação pós-graduada brasileira com ênfase na área de planejamento regional e urbano. Os pesquisadores realçam que essa modalidade stricto sensu contribui para atender a crescente demanda por qualificação gerada pelas questões relativas ao desenvolvimento regional, descentralização administrativa e entrada em vigor do Estatuto da Cidade e da Lei de Responsabilidade Fiscal;

- Quelhas, Faria Filho e França (2005) discutem o papel desempenhado pelos mestrados profissionais no sistema de pós-graduação do Brasil. Este trabalho reúne uma abordagem crítica, definições e discussão sobre a importância dos programas de mestrado profissional na interação entre os problemas profissionais e o conhecimento produzido na universidade;

- Belloni (2000) modela a avaliação do desempenho de universidades federais brasileiras através do uso interativo de técnicas estatísticas e de análise envoltória de dados (Data Envelopment Analysis - DEA). A modelagem considera indicadores da pós-graduação e pesquisa, e um indicador da graduação;

- Carvalho (2001) discute critérios para avaliação dos programas de pós-graduação com base em um levantamento sobre os primeiros 25 anos do curso de pós-graduação de Psicologia Experimental da USP. Essa pesquisa recomenda a autoavaliação como ferramenta de validação e questionamento de avaliações externas e propõe a utilização dos seguintes critérios para avaliação dos programas: participação dos alunos na produção científica, avaliação do curso pelos alunos e destino profissional dos titulados;

- Sant'anna (2002) compara três métodos de agregação de critérios da produtividade global: DEA clássica (CHARNES; COOPER; RHODES, 1978); produtividade global avaliada probabilisticamente (SANT'ANNA, 2001); e produtividade explicada por um modelo hierárquico (SANT'ANNA, 1999). A pesquisa investiga o impacto da variação da quantidade de alunos em cursos de pós-graduação em Engenharia de Produção em dois quesitos da produtividade docente: formação de mestres e geração de resultados de pesquisa. A evolução dessa pesquisa está reportada em Sant'anna (2005);

- Lins, Almeida e Bartholo Junior (2004) destacam o uso de DEA como apoio quantitativo para subsidiar a interpretação da avaliação de programas de pós-graduação. A abordagem proposta foi aplicada aos programas de Engenharia de Produção reconhecidos pela Capes;

- Moreira, Hortale e Hartz (2004) discutem possíveis modificações no sistema de avaliação da pós-graduação no Brasil. As propostas são realizadas mediante pesquisa de opinião junto ao colegiado de doutores da Fundação Oswaldo Cruz sobre proposições sugeridas pelos convidados internacionais da Capes na avaliação de 2001;

- Spagnolo e Souza (2004) abordam a composição dos critérios de avaliação, adotados no sistema de avaliação da Capes. Na pesquisa, realizada durante a avaliação de 2004, foram consultados os membros das comissões de avaliação e coordenadores de programas de pós-graduação de instituições privadas sobre a oportunidade de implementação de sugestões formuladas pela comunidade acadêmica a respeito do sistema de avaliação. Essa pesquisa também considerou aspectos da avaliação de mestrados profissionais;

- Cordeiro e Parente (2004) apresentam uma proposta para identificação do perfil profissional e acadêmico dos egressos dos cursos de pós-graduação stricto sensu da Universidade Federal do Amazonas (UFAM) e para avaliação da qualidade dos serviços prestados pela instituição. Foi utilizada a estatística descritiva para analisar os dados e formular propostas para melhoria do ensino oferecido pela instituição;

- Kerr-Pontes et al. (2005) refletem sobre os critérios utilizados pela Capes na avaliação da pós-graduação, com destaque para os programas da área de Saúde Coletiva. A pesquisa considera a contribuição dos processos avaliativos na qualidade do sistema de ensino e pesquisa. Em contrapartida, aponta o impacto produzido pelas concepções de ciência, 
subjacentes à formulação dos critérios, e os obstáculos encontrados na avaliação de cursos que desenvolvem suas práticas em contextos distintos;

- Almeida Filho e Ramos (2005) usam os critérios adotados pela Capes para a avaliação dos programas de pós-graduação em Engenharia de Produção das instituições federais de ensino superior (IFES). A pesquisa utilizou DEA para relacionar múltiplas variáveis numa avaliação quantitativa dos critérios;

- Rocha (2006) elabora proposta para autoavaliação do Centro de Pós-Graduação da Fundação Visconde de Cairu. A metodologia baseia-se na participação da comunidade acadêmica, através de oficinas coletivas para construção do diagnóstico institucional. Os resultados apontam a ação articulada entre avaliação e gestão organizacional como instrumento para a qualidade institucional;

- Miranda e Almeida (2003) investigaram a aplicação do método ELECTRE TRI, que é um método de auxílio multicritério à decisão (AMD), à avaliação de programas de pós-graduação (stricto sensu). Esse trabalho toma por objeto de estudo o sistema de avaliação da Capes e os resultados da avaliação de curso da área "Engenharias 111 " da Capes. Seguindo essa linha de pesquisa, Miranda e Almeida (2004) investigam a ordenação (ou ranking) de programas de curso da área "Engenharias 111 " da Capes. Mais especificamente o trabalho intercompara os resultados obtidos pelo emprego de dois diferentes métodos multicritério ao problema de ordenação: o ELECTRE 11 e o MAUT; e

- Ainda no contexto da avaliação da pós-graduação, Neves (2005) apresenta um caso específico de avaliação e classificação de desempenho e posicionamento estratégico de um programa de mestrado em Engenharia de Produção. Essa pesquisa investiga a integração da análise SWOT ao ELECTRE TRl. Extensões desse trabalho são encontradas em Neves e Costa (2006).

Seguindo a linha da aplicação do AMD à avaliação de instituições de ensino, encontram-se os trabalhos de:

- Costa e Freitas (2000), que propõem a aplicação do ELECTRE TRI à avaliação do desempenho de docentes em sistemas de ensino, classificando-os em categorias ou classes de desempenho. Esse trabalho foi estendido nos trabalhos de Motta (2002) e Barroso (2002) que adotaram, respectivamente, os métodos ELECTRE 11 e 111 para estabelecer o ranking de docentes segundo critérios de produtividade dos mesmos;

- Mello, Gomes e Lins (2002), que utilizam o método MACBETH para avaliação da presença e/ou visibilidade da Universidade Federal Fluminense nos municípios com presença desta instituição no processo de interiorização universitária;
- Ribeiro (2003), que apresenta uma modelagem para a classificação da percepção do desempenho de IES, sob a ótica discente, com emprego do método ELECTRE TRl. Esse trabalho considera a avaliação em diferentes contextos (Ciência da Computação, Ciências Contábeis, Direito, Administração e Economia), em diferentes intervalos de tempo. Os resultados obtidos nessa pesquisa estão também publicados em Ribeiro e Costa (2005);

- Politis e Siskos (2004), que se baseiam no método MUSA (Multicriteria Satisfaction Analysis - Grigoroudis e Siskos (2001)) para avaliação do desempenho do Departamento de Engenharia de Produção e Gestão da Universidade Técnica de Creta. Os critérios considerados nessa modelagem tratam aspectos ligados a três diretrizes: operação e processos internos, satisfação dos estudantes e aceitação pelo mercado de trabalho. Ainda segundo esses autores, as universidades, como instituições de ensino e pesquisa, precisam ser constantemente avaliadas, para que possam preparar a sociedade para as mudanças geradas pela globalização e pela reestruturação do mercado de trabalho;

- Meca (2006), que investiga a aplicação de diferentes métodos de AMD (ELECTRE 1, ELECTRE 11, CONDORCET e BORDA) à avaliação de instituições de ensino secundário, comparando os resultados obtidos com aqueles fornecidos pelo Programa Nova Escola (programa oficial de avaliação das escolas secundárias no estado do Rio de Janeiro). Esse estudo foca a avaliação de escolas secundárias do município de Macaé, situado na região da Bacia de Campos; e

- Freitas, Rodrigues e Costa (2009), que propõem uma abordagem multicritério fundamentada no tradicional método da média ponderada para classificar o desempenho de instituições de ensino superior (IES) segundo percepções de professores e alunos. Os critérios utilizados na avaliação foram agrupados em três dimensões: organização institucional, corpo docente/corpo discente e instalações.

A metodologia proposta no presente trabalho baseia-se na abordagem apresentada em Costa e Costa (2003), que avaliam as diferenças entre as percepções dos estudantes de uma IES e os gestores dessa mesma instituição. Este estudo foi baseado numa adaptação do modelo de lacunas (PARASURAMAN; ZEITHAML; BERRY, 1985) e também foi explorado em:

- Ribeiro (2004), que desenvolve um trabalho de mapeamento das lacunas existentes entre as percepções dos laboratórios de ensaios credenciados no setor de construção civil e da Coordenação Geral de Credenciamento (CGCRE) do Inmetro sobre os impactos oriundos do credenciamento desses laboratórios. A proposta permite identificar lacunas nas percepções intraorganizacionais da CGCRE, 
servindo como ferramenta de gestão na busca de atendimento aos anseios dos clientes externos;

- Nogueira (2004), que apresenta uma análise dos impactos gerados pela implantação e prática do sistema de gerenciamento da rotina na área de manutenção pesada de uma empresa de transporte aéreo. Utiliza abordagem baseada no modelo de lacunas que permite mapear e comparar as percepções dos gestores e empregados envolvidos no processo; e

- Costa (2005), que propõe um modelo para mapeamento das lacunas de percepção entre as empresas que utilizam a solução e-procurement e uma das maiores empresas que disponibilizam a solução e-procurement no mercado brasileiro. Os resultados obtidos nesta pesquisa estão também publicados em Costa e Costa (2007).

\section{Metodologia}

A metodologia adotada tem por base o trabalho de Costa e Costa (2003) e, como referência inicial para a construção do conjunto de critérios e elaboração do instrumento de coleta, o trabalho de Neves (2005) - a qual foi aprimorada pela consideração de informações coletadas e registradas na seção Revisão bibliográfica deste trabalho.

\subsection{Etapas da metodologia}

A abordagem proposta está estruturada no desenvolvimento das seguintes etapas:

(a) Caracterizar o programa sob avaliação;

(b) Especificar os critérios que serão considerados;

(c) Especificar a escala construída para o julgamento dos critérios;

(d) Coletar os julgamentos de valor;

(e) Analisar os resultados obtidos junto aos alunos;

(f) Analisar os resultados obtidos junto aos chefes; e

(g) Comparar as percepções dos dois grupos.

\subsection{Caso de aplicação da proposta}

Nesta seção apresenta-se a aplicação da abordagem proposta a um caso real.

(a) Caracterizar o programa sob avaliação. Os dados foram coletados no contexto de uma turma específica de um determinado curso de mestrado na área de Engenharias 111.

A coleta de dados foi aplicada a um universo restrito composto por apenas uma das turmas de um determinado programa de mestrado profissional. Ainda que a representatividade dos resultados obtidos da análise do caso específico implique em restrição e limitação, isso não prejudica a generalidade da metodologia proposta.

A escolha da turma participante da pesquisa foi motivada pela conveniência de acesso aos dados. Foi escolhida uma turma do Programa de Mestrado Profissional em Sistemas \& Gestão (MSG) que está ligado à Escola de Engenharia, Departamento de Engenharia de Produção da Universidade Federal Fluminense (UFF), classificado na área de Engenharias 111 da Capes, para mapeamento das percepções discentes.

Para melhor compreensão do contexto que envolve a pesquisa, destacam-se:

- 0 MSG/UFF foi criado em 2000 e tem como objetivo levar os seus egressos à reflexão sobre a aderência dos modelos organizacionais aos resultados, sem deixar de enfatizar as preocupações com a humanização, atuação responsável, ética e impactos no meio ambiente. A disputa pelo ingresso no curso configurava-se em torno de 2,7 alunos/vaga, e no período desta pesquisa o curso contava com aproximadamente 220 alunos matriculados. A turma pesquisada pode ser considerada como a $4^{a}$ turma a ingressar no curso;

- Para obter a avaliação nas disciplinas do curso, os alunos devem comprovar proficiência nos conteúdos das disciplinas cursadas através de exames escritos. Como estratégia de ensino e desenvolvimento do egresso é usual que, nas disciplinas, os alunos sejam convocados a apresentar trabalhos individuais e em grupos. Também é demandada desse aluno a sua participação em seminários e workhops. Outra característica do curso é o estímulo ao desenvolvimento de pesquisas aplicadas à solução de problemas no contexto organizacional. A solução de problemas organizacionais é uma prática também constante no contexto do desenvolvimento do itinerário acadêmico ao longo das disciplinas do curso; e

- Assim como em outros programas de pós-graduação stricto sensu no Brasil, os alunos desse curso devem defender dissertação de mestrado perante banca examinadora.

Os participantes da pesquisa podem ser divididos da seguinte maneira:

- Grupo 1: Alunos de uma turma do curso de mestrado profissional do MSG/UFF (composto por 33 discentes); e

- Grupo 2: Chefes desses alunos na empresa onde trabalham (composto por 5 chefes).

Vale registrar que neste trabalho: 
- A palavra "aluno" é usada indiscriminadamente para discentes e egressos do curso (sendo que os discentes, em sua totalidade, já haviam concluído os créditos e encontravam-se em fase de conclusão da dissertação - ou já haviam defendido a mesma); e

- A palavra "chefe" é adotada para definir os membros de uma empresa que representavam os chefes diretos dos alunos do mestrado profissional.

Os questionários foram encaminhados por correio eletrônico aos alunos e chefes. A Tabela 1 apresenta as porcentagens das repostas obtidas em função dos questionários enviados.

Tabela 1. Percentagens de respostas aos questionários enviados.

\begin{tabular}{lccc}
\hline & $\begin{array}{c}\text { Questionários } \\
\text { enviados }\end{array}$ & Respondentes & $\begin{array}{c}\text { Percentual de } \\
\text { respostas (\%) }\end{array}$ \\
\hline Grupo 1 (alunos) & 33 & 21 & $63,64 \%$ \\
Grupo 2 (chefes) & 5 & 4 & $80 \%$ \\
\hline
\end{tabular}

(b) Especificação dos critérios considerados. Os critérios adotados foram definidos com base na revisão bibliográfica. 0 Quadro 1 apresenta os 29 critérios utilizados no questionário para coletar impressões junto aos alunos. 0 Quadro 2 apresenta os 16 critérios utilizados para pesquisar as percepções dos chefes. Quinze questões, comuns aos dois questionários, serviram de base na análise das lacunas (diferenças) de percepção entre alunos e chefes;

(c) Especificação da escala construída para o julgamento dos critérios. Dois aspectos foram pesquisados: desempenho e importância. As escalas de julgamento estão baseadas em Likert (1932), Miller (1954) e Costa et al. (2007) e encontram-se descritas nos Quadros 3 e 4;

(d) Coleta dos julgamentos de valor. Os questionários foram distribuídos por correio eletrônico para 33 alunos (total de alunos da turma) e 5 chefes no dia 11 de julho de 2006. As respostas foram obtidas em uma semana, com respostas de 21 alunos

Quadro 1. Critérios utilizados na pesquisa com os alunos.

\begin{tabular}{|c|c|c|}
\hline Código & Critério & Descrição do critério \\
\hline $\mathrm{C}_{1}$ & Impacto na empregabilidade & Impacto na empregabilidade do pós-graduado. \\
\hline$C_{2}$ & Nível de resposta às expectativas da empresa & $\begin{array}{l}\text { Correlação da grade curricular com as reais necessidades encontradas na } \\
\text { empresa. }\end{array}$ \\
\hline $\mathrm{C}_{3}$ & Remuneração do pós-graduado & Impacto na remuneração do pós-graduado. \\
\hline $\mathrm{C}_{4}$ & Perfil empreendedor & Impacto no perfil de empreendedor do pós-graduado. \\
\hline $\mathrm{C}_{5}$ & Relacionamento interpessoal & Impacto na capacidade do pós-graduado para trabalhar em equipe. \\
\hline $\mathrm{C}_{6}$ & Perfil pesquisador & Impacto no perfil de pesquisador do pós-graduado. \\
\hline $\mathrm{C}_{7}$ & Perfil negociador & Impacto no perfil de negociador do pós-graduado. \\
\hline $\mathrm{C}_{8}$ & Autoestima & Impacto na autoestima do pós-graduado. \\
\hline $\mathrm{C}_{9}$ & Oratória & Impacto na capacidade, do pós-graduado, de falar em público. \\
\hline $\mathrm{C}_{10}$ & Vida pessoal & Impacto na vida pessoal do estudante. \\
\hline $\mathrm{C}_{11}$ & Desinibição & Influência no desembaraço do pós-graduado. \\
\hline $\mathrm{C}_{12}$ & Capacidade de expressão & $\begin{array}{l}\text { Impacto na capacidade de expressão do pós-graduado (exposição de ideias, } \\
\text { sugestões, conceitos, conclusões etc.). }\end{array}$ \\
\hline $\mathrm{C}_{13}$ & Senso crítico & Impacto na capacidade do estudante em estabelecer críticas. \\
\hline $\mathrm{C}_{14}$ & Absorção de críticas & Impacto na capacidade do estudante em receber críticas. \\
\hline $\mathrm{C}_{15}$ & Solução de problemas & Impacto na capacidade do estudante em resolver problemas. \\
\hline$C_{16}$ & Uso de métodos organizados & Impacto na capacidade de aplicação de métodos organizados. \\
\hline $\mathrm{C}_{17}$ & Número de professores & Número de professores permanentes no programa. \\
\hline $\mathrm{C}_{18}$ & Proporção entre estudante e professor & Proporção de número de estudantes por professor. \\
\hline $\mathrm{C}_{19}$ & Assistência ao estudante & Serviços de secretaria. \\
\hline $\mathrm{C}_{20}$ & Material Didático (livros, notas de aula etc.) & Qualidade do material didático. \\
\hline $\mathrm{C}_{21}$ & Material didático (livros, notas de aula etc.) & Suficiência do material didático. \\
\hline $\mathrm{C}_{22}$ & Infraestrutura disponível & Disponibilidade de equipamentos tecnológicos e computadores. \\
\hline $\mathrm{C}_{23}$ & Softwares disponíveis & Disponibilidade e uso de softwares. \\
\hline $\mathrm{C}_{24}$ & Instalações físicas adequadas & Adequação dos prédios utilizados. \\
\hline $\mathrm{C}_{25}$ & Biblioteca & Adequação das bibliotecas às necessidades dos estudantes. \\
\hline $\mathrm{C}_{26}$ & Salas de estudo & Adequação das salas de estudo. \\
\hline $\mathrm{C}_{27}$ & Desenvolvimento das disciplinas & Qualidade das disciplinas oferecidas (teoria e prática). \\
\hline$C_{28}$ & $\begin{array}{l}\text { Adequação das disciplinas à proposta do } \\
\text { programa }\end{array}$ & $\begin{array}{l}\text { Associação entre o desenvolvimento das disciplinas e os objetivos do } \\
\text { programa. }\end{array}$ \\
\hline $\mathrm{C}_{29}$ & Programa das disciplinas & Programação das disciplinas, sequência e abordagem dos capítulos. \\
\hline
\end{tabular}


e 4 chefes - o que demonstra o interesse da amostra na pesquisa. A compilação das respostas obtidas está reportada nos Anexos 1 e 2; e

(e) Análise dos resultados obtidos junto aos alunos. As Figuras 1 e 2 apresentam os valores médios das percepções dos alunos quanto ao desempenho do programa e à importância dos critérios.
Da Figura 1, destaca-se que não houve questão com valor médio negativo. 0 menor valor $(0,1)$ refere-se aos critérios $C_{3}$ e $C_{25}$ que versam, respectivamente, sobre o impacto na remuneração dos alunos e adequação das bibliotecas. Os maiores valores médios de desempenho, 1,7 $\left(\mathrm{C}_{6}\right)$ e 1,6 $\left(\mathrm{C}_{8}\right.$ e $\left.\mathrm{C}_{13}\right)$, versam sobre o impacto no perfil pesquisador, na autoestima e no senso crítico do pós-graduado.

Quadro 2. Critérios utilizados na pesquisa com os chefes.

\begin{tabular}{|c|c|c|}
\hline Código & Critério & Descrição do critério \\
\hline$C_{1}$ & Material didático (livros, notas de aula etc.) & Qualidade do material didático. \\
\hline $\mathrm{C}_{2}$ & Material didático (livros, notas de aula etc.) & Suficiência do material didático. \\
\hline $\mathrm{C}_{3}$ & Negociação com a coordenação do curso & $\begin{array}{l}\text { Negociação dos representantes da empresa com a coordenação do } \\
\text { curso. }\end{array}$ \\
\hline $\mathrm{C}_{4}$ & Perfil empreendedor & Impacto no perfil de empreendedor do pós-graduado. \\
\hline $\mathrm{C}_{5}$ & Perfil pesquisador & Impacto no perfil de pesquisador do pós-graduado. \\
\hline $\mathrm{C}_{6}$ & Perfil negociador & Impacto no perfil de negociador do pós-graduado. \\
\hline $\mathrm{C}_{7}$ & Relacionamento interpessoal & Impacto na capacidade do pós-graduado para trabalhar em equipe. \\
\hline $\mathrm{C}_{8}$ & Nível de resposta às necessidades da companhia & $\begin{array}{l}\text { Correlação da grade curricular com as reais necessidades encontradas } \\
\text { na empresa. }\end{array}$ \\
\hline $\mathrm{C}_{9}$ & Autoestima & Impacto na autoestima do pós-graduado. \\
\hline $\mathrm{C}_{10}$ & Oratória & Impacto na capacidade, do pós-graduado, de falar em público. \\
\hline $\mathrm{C}_{11}$ & Desinibição & Influência no desembaraço do pós-graduado. \\
\hline $\mathrm{C}_{12}$ & Capacidade de expressão & $\begin{array}{l}\text { Impacto na capacidade de expressão do pós-graduado (exposição de } \\
\text { ideias, sugestões, conceitos, conclusões etc.). }\end{array}$ \\
\hline $\mathrm{C}_{13}$ & Senso crítico & Impacto na capacidade em estabelecer críticas. \\
\hline $\mathrm{C}_{14}$ & Absorção de críticas & Impacto na capacidade do estudante em receber críticas. \\
\hline$C_{15}$ & Solução de problemas & Impacto na capacidade do estudante em resolver problemas. \\
\hline $\mathrm{C}_{16}$ & Uso de métodos organizados & Impacto na capacidade de aplicação de métodos organizados. \\
\hline
\end{tabular}

Fonte: os autores

Quadro 3. Escala para julgamento do grau de desempenho.

\begin{tabular}{cccccc}
\hline Muito bom & Bom & Regular & Ruim & Muito ruim & Não sei opinar \\
\hline 2 & 1 & 0 & -1 & -2 & $\mathrm{~N}$ \\
\hline
\end{tabular}

Fonte: os autores

Quadro 4. Escala para julgamento do grau de importância.

\begin{tabular}{cccccc}
\hline Muito importante & Importante & Importância média & Pouco importante & Nada importante & Não sei opinar \\
\hline 4 & 3 & 2 & 1 & 0 & $\mathrm{~N}$ \\
\hline
\end{tabular}

Fonte: os autores

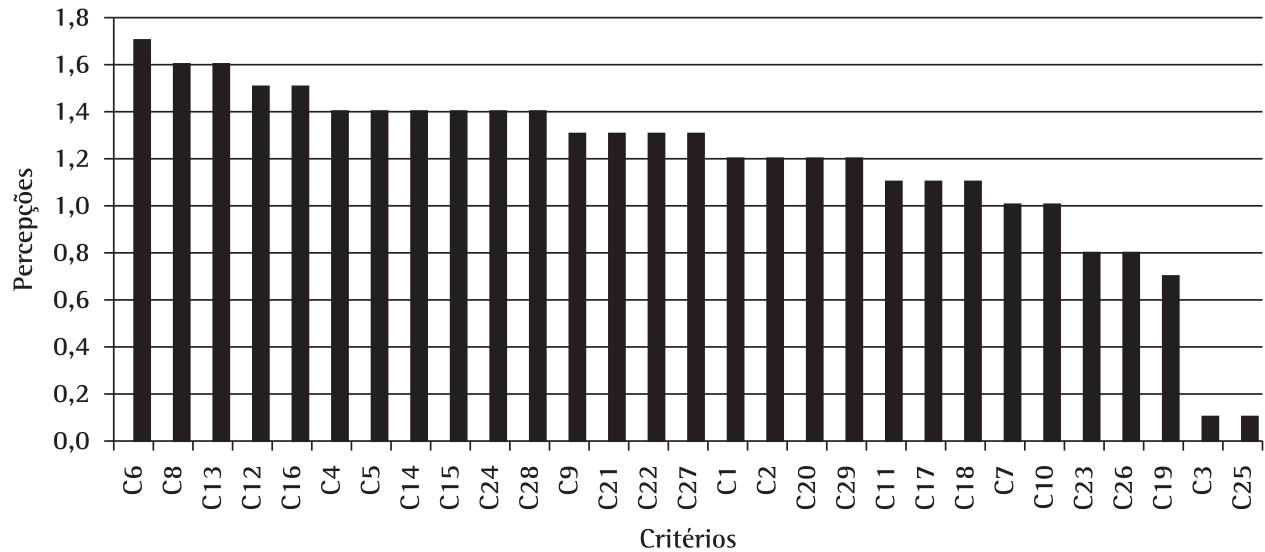

Figura 1. Percepções dos alunos sobre o nível de desempenho em ordem decrescente. Fonte: os autores. 
Da Figura 2 nota-se que o critério $\mathrm{C}_{11}$ (desinibição dos estudantes) obteve o menor valor médio de importância $(2,9)$; e o critério qualidade das disciplinas $\left(C_{27}\right)$ foi considerado o mais importante $(3,9)$.

Com relação às percepções dos alunos ainda destaca-se que:

- Os critérios C3 (impacto na remuneração do pós-graduado) e C25 (adequação das bibliotecas) apresentaram alto grau de importância e o menor grau de desempenho;

- 0 critério $C_{27}$ (desenvolvimento das disciplinas) registrou, simultaneamente, maior nível de importância, e nível de desempenho igual a 1,3; e

- 0 impacto no perfil pesquisador $\left(\mathrm{C}_{6}\right)$ obteve maior grau de desempenho e também aparece entre os critérios mais importantes com valor médio igual a 3,8.

(f) Análise dos resultados obtidos junto aos chefes. Os valores médios das percepções dos chefes, quanto ao desempenho e importância em cada critério avaliado, são apresentados nas Figuras 3 e 4.

Da Figura 3, observa-se que a questão $\mathrm{C}_{3}$, que aborda a negociação da empresa com a coordenação do curso, obteve menor valor médio de desempenho $(0,5)$. A maior média $(1,8)$ foi obtida em metade dos critérios relacionados no questionário, descritos a seguir:

- $C_{1}$. Qualidade do material didático;

- $\mathrm{C}_{5}$ Impacto no perfil pesquisador dos estudantes;

- $\mathrm{C}_{7}$ Impacto na capacidade de trabalhar em equipe;

- $\mathrm{C}_{8}$ - Nível de resposta às necessidades da companhia;

- $C_{9}$ Impacto na autoestima do pós-graduado;

- $C_{12}$ Impacto na capacidade de expressão do pós-graduado;

- $\mathrm{C}_{15}$ - Impacto na capacidade em resolver problemas; e

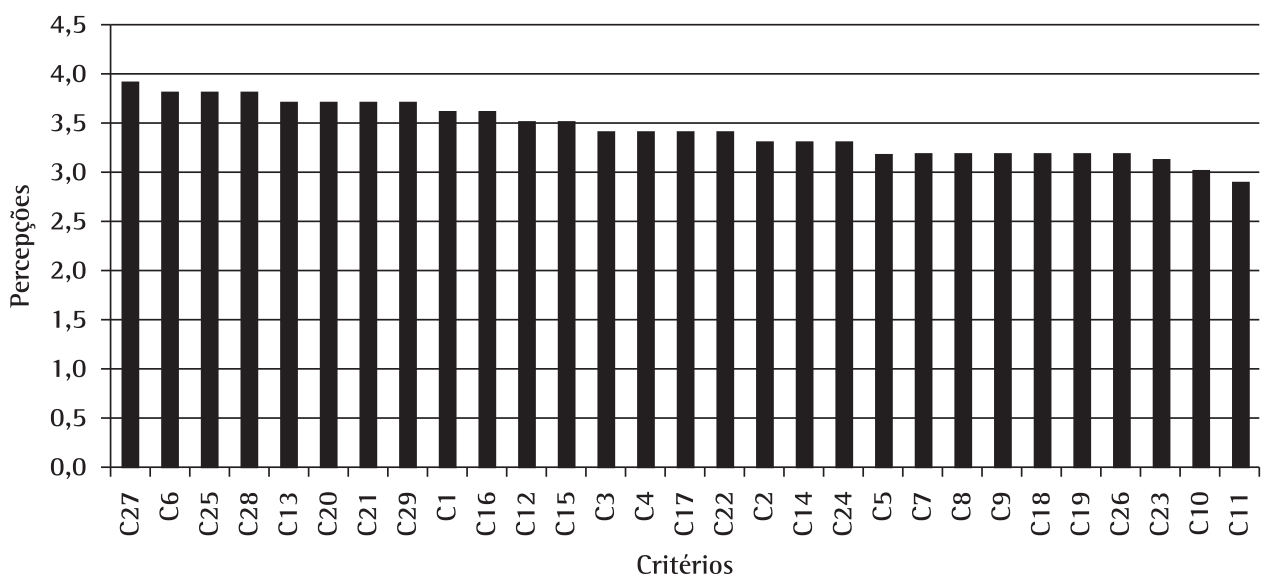

Figura 2. Percepções dos alunos sobre o nível de importância em ordem decrescente. Fonte: os autores.

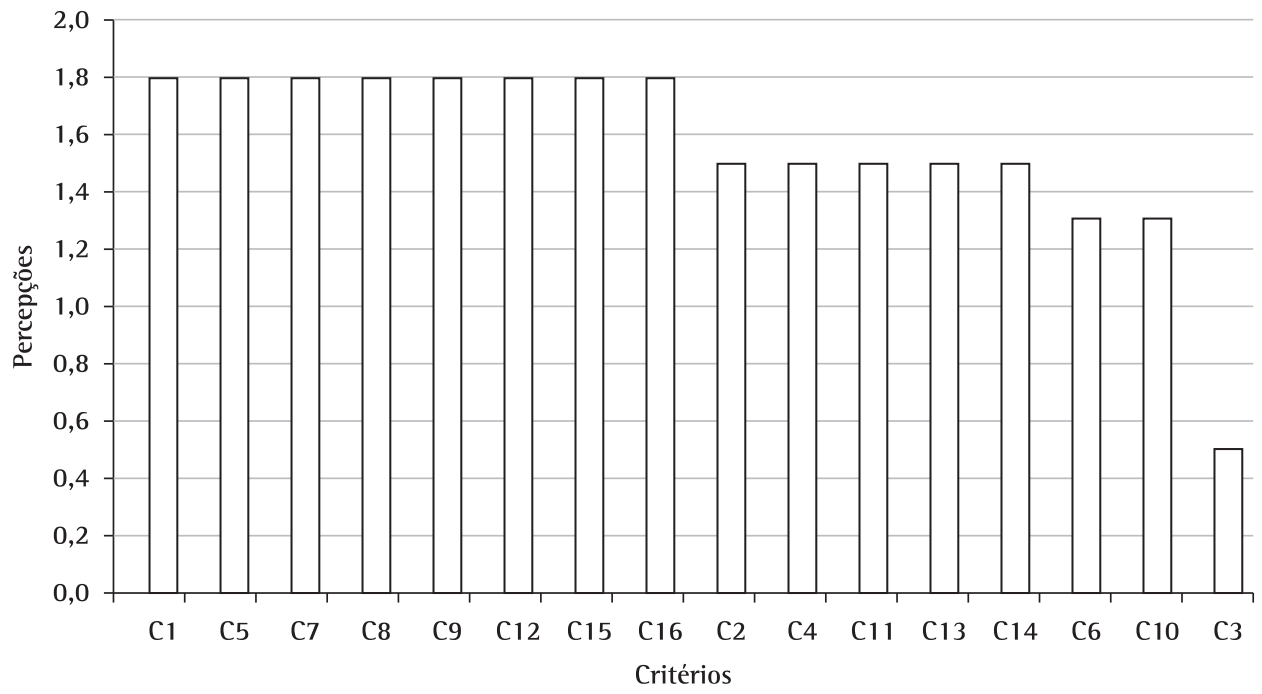

Figura 3. Percepções dos chefes sobre o nível de desempenho em ordem decrescente. Fonte: os autores. 
- $\mathrm{C}_{16-}$ Capacidade de aplicação de métodos organizados.

Da Figura 4 observa-se que o menor valor $(3,0)$ foi encontrado em dois quesitos: $C_{2}$ (suficiência do material didático) e $\mathrm{C}_{3}$ (negociação com a coordenação do curso). Três questões merecem maior atenção, pois receberam valor máximo de importância na visão dos quatro chefes, são elas: $C_{7}$ (impacto na capacidade do pós-graduado em trabalhar em equipe), $C_{15}$ (impacto na capacidade do estudante em resolver problemas) e $\mathrm{C}_{16}$ (impacto na capacidade de aplicação de métodos organizados).

Sobre as percepções dos chefes destaca-se que:

- Os critérios $\mathrm{C}_{7}$ (relacionamento interpessoal), $\mathrm{C}_{15}$ (capacidade em resolver problemas) e $\mathrm{C}_{16}$ (uso de métodos organizados) apresentaram maior grau de importância e encontram-se entre os que obtiveram maior desempenho;

- A questão $C_{3}$ (negociação com a coordenação do curso) apresentou o menor nível de desempenho, e também menor grau de importância; e

- Os aspectos $\mathrm{C}_{1}, \mathrm{C}_{5}, \mathrm{C}_{7}, \mathrm{C}_{8}, \mathrm{C}_{9}, \mathrm{C}_{12}, \mathrm{C}_{15}, \mathrm{C}_{16}$, com valor médio de desempenho igual a 1,8 , encontram-se entre os mais importantes na visão dos chefes.

(g) Comparação entre as percepções dos dois grupos. As lacunas são identificadas quando comparamos percepções de diferentes grupos de pessoas, sobre um mesmo critério ou grupo de critérios. Esta pesquisa captou percepções de dois grupos distintos (alunos e chefes). Os critérios comuns aos dois questionários, apresentados no Quadro 5,

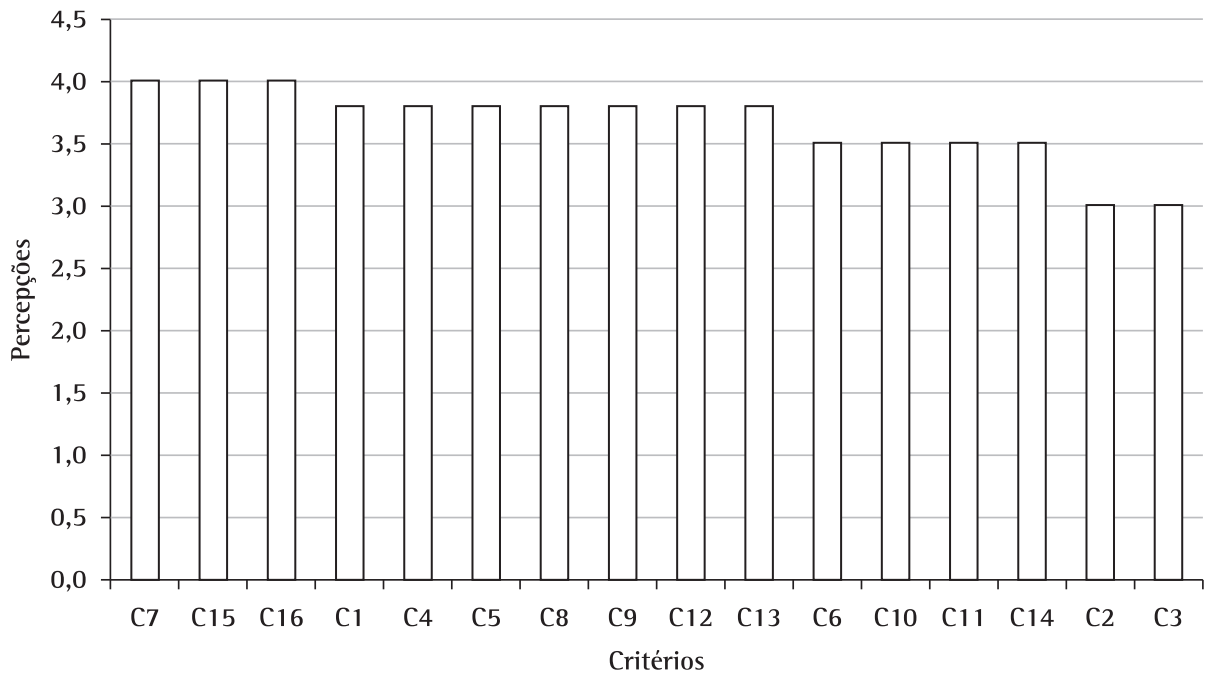

Figura 4. Percepções dos chefes sobre o nível de importância em ordem decrescente. Fonte: os autores.

Quadro 5. Critérios comuns na pesquisa dos dois grupos.

\begin{tabular}{cll}
\hline Código & \multicolumn{1}{c}{ Critério } & \multicolumn{1}{c}{ Descrição do critério } \\
\hline $\mathrm{L}_{1}$ & Material didático (livros, notas de aula etc.) & Qualidade do material didático. \\
$\mathrm{L}_{2}$ & Material didático (livros, notas de aula etc.) & Suficiência do material didático. \\
$\mathrm{L}_{3}$ & Perfil empreendedor & Impacto no perfil de empreendedor do pós-graduado. \\
$\mathrm{L}_{4}$ & Perfil pesquisador & Impacto no perfil de pesquisador do pós-graduado. \\
$\mathrm{L}_{5}$ & Perfil negociador & Impacto no perfil de negociador do pós-graduado. \\
$\mathrm{L}_{6}$ & Relacionamento interpessoal & Impacto na capacidade do pós-graduado para trabalhar em equipe. \\
$\mathrm{L}_{7}$ & Nível de resposta às expectativas da empresa & Correlação da grade curricular com as reais necessidades encontradas na \\
& empresa. \\
$\mathrm{L}_{8}$ & Autoestima & Impacto na autoestima do pós-graduado. \\
$\mathrm{L}_{9}$ & Oratória & Impacto na capacidade, do pós-graduado, de falar em público. \\
$\mathrm{L}_{10}$ & Desinibição & Influência no desembaraço do pós-graduado. \\
$\mathrm{L}_{11}$ & Capacidade de expressão & Impacto na capacidade de expressão do pós-graduado (exposição de ideias, \\
$\mathrm{L}_{12}$ & Senso crítico & sugestões, conceitos, conclusões etc.). \\
$\mathrm{L}_{13}$ & Absorção de críticas & Impacto na capacidade do estudante em estabelecer críticas. \\
$\mathrm{L}_{14}$ & Solução de problemas & Impacto na capacidade do estudante em receber críticas. \\
$\mathrm{L}_{15}$ & Uso de métodos organizados & Impacto na capacidade do estudante em resolver problemas. \\
\hline
\end{tabular}


juntamente com as lacunas (diferenças) entre os valores médios das percepções dos alunos e chefes, estão ilustrados nas Figuras 5 e 6.

Da Figura 5 destaca-se que:

- As principais diferenças de percepção quanto ao desempenho ocorreram nas lacunas $\mathrm{L}_{1}$ (qualidade do material didático) e $\mathrm{L}_{7}$ (nível de resposta do curso às necessidades da empresa);
- Há harmonização de percepções sobre os efeitos impactantes do curso na capacidade de falar em público dos estudantes $\left(\mathrm{L}_{9}\right)$;

- De forma geral, o curso apresenta maior desempenho na visão dos chefes. Apenas na lacuna $\mathrm{L}_{12}$ (impacto na capacidade em estabelecer críticas) observa-se maior valor percebido pelos alunos quando comparados às percepções dos chefes;

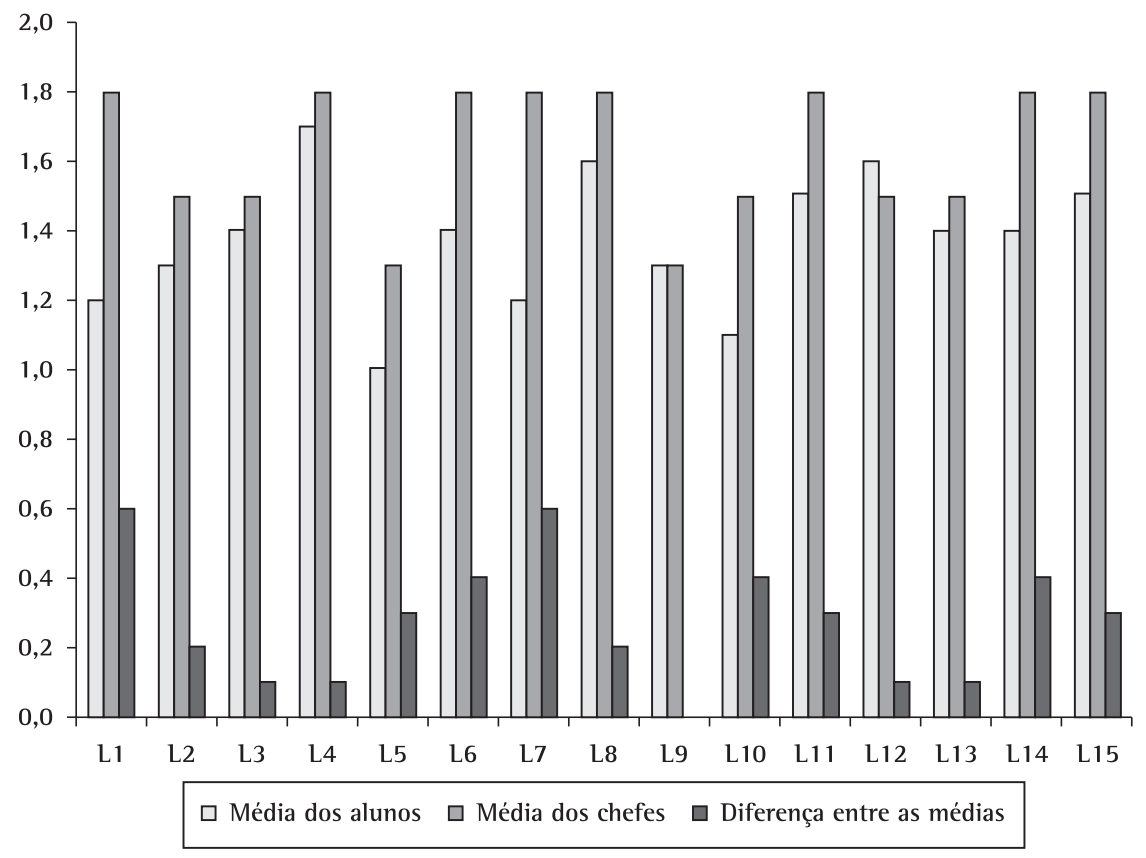

Figura 5. Lacunas de percepção: desempenho. Fonte: os autores.

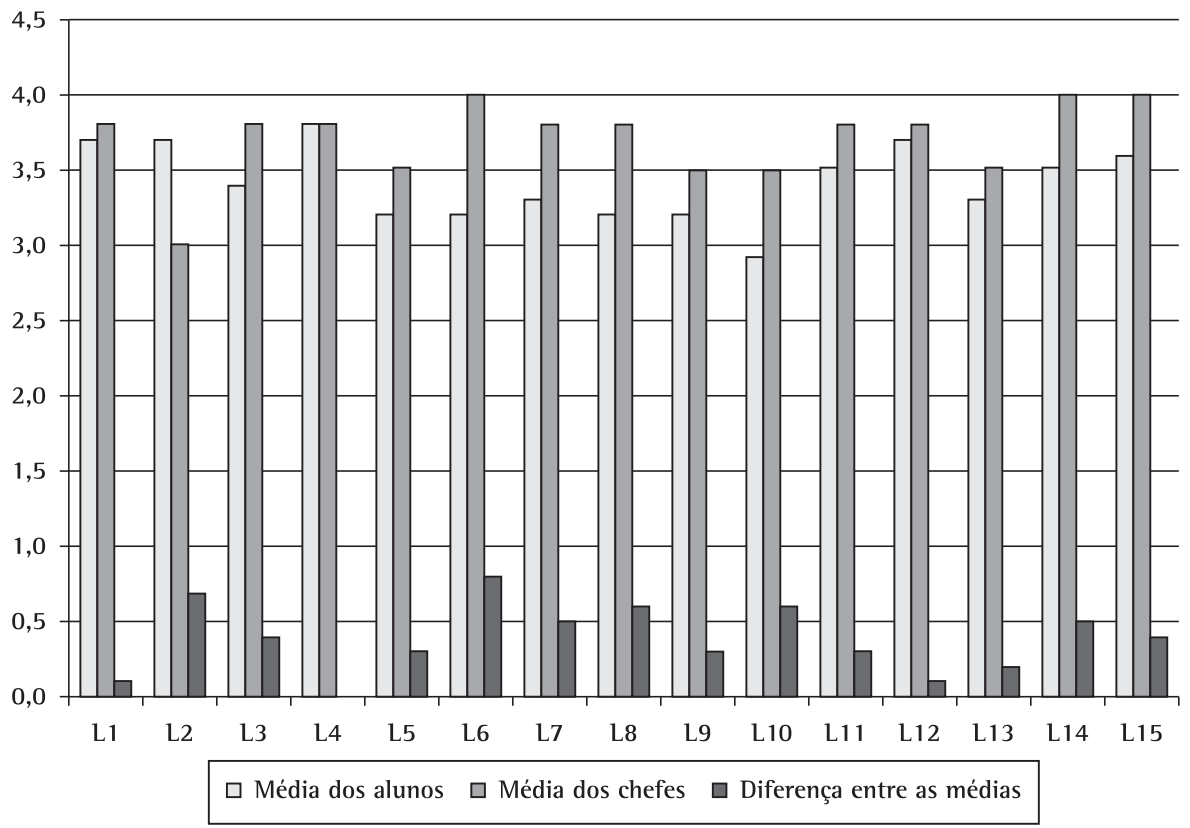

Figura 6. Lacunas de percepção: importância. Fonte: os autores. 
- A lacuna $\mathrm{L}_{4}$ (associada ao questão perfil pesquisador) indica que esse é aspecto percebido com maior grau de desempenho na visão dos alunos e também na percepção dos chefes; e

- $\mathrm{Al}_{5}$ (associada ao perfil negociador) indica que houve uma aproximação entre as percepções dos discentes e dos chefes: ambos perceberam essa característica como a menos influenciado pelo curso.

A Figura 6 mostra que as principais lacunas de percepção de importância foram: $\mathrm{L}_{2}$ (suficiência do material didático) e $\mathrm{L}_{6}$ (relacionamento interpessoal). $A$ harmonização de percepções em $\mathrm{L}_{4}$ indica que tanto os alunos quanto os chefes consideram relevante 0 desenvolvimento do perfil pesquisador do egresso. A lacuna $\mathrm{L}_{15}$ (uso de métodos organizados) indica que este aspecto foi considerado o mais importante em ambas as visões: chefes e alunos. Observando as quinze lacunas estudadas, a $\mathrm{L}_{2}$ foi a única em que a importância percebida pelos discentes foi superior à dos chefes. Ou seja: o aluno atribui maior importância à suficiência do material didático.

\section{Conclusões}

A carência identificada na literatura, de textos que tratam desse tema, somada à novidade que representa o mestrado profissional no Brasil e à abordagem metódica utilizada na pesquisa amplificam o grau de originalidade e a contribuição deste trabalho.

A pesquisa efetuada para experimentação da proposta deste artigo objetivou o mapeamento de percepções, o qual foi efetuado com "discentes-funcionários" da organização e seus respectivos chefes. Essa abordagem foi facilitada pelo fato de que todos os pesquisados estavam na organização nos períodos imediatamente anterior ao início do curso e posterior à conclusão deste, sendo considerados aptos a manifestarem as suas percepções.

$\mathrm{Na}$ construção da pesquisa foram adotados escalas específicas para o tratamento de problemas de julgamentos de valor e questionário elaborado a partir de aspectos garimpados na extensa revisão bibliográfica realizada. Esses dois aspectos contribuem significativamente para a credibilidade dos resultados obtidos na pesquisa, destacando-se que, além dos aspectos associados ao desempenho acadêmico, o questionário considerou questões relacionadas ao desempenho profissional do egresso.

Quanto aos resultados da pesquisa, destaca-se que:

- Nenhuma questão apresentou valor médio de importância inferior a 2,9. Isso confirma a relevância dos critérios adotados no questionário;

- Em geral, os valores médios de importância e desempenho atribuídos pelos chefes foram maiores que os valores atribuídos pelos alunos;
- Na percepção dos alunos, dentre os critérios considerados mais importantes $\left(\mathrm{C}_{27}, \mathrm{C}_{6}, \mathrm{C}_{16}, \mathrm{C}_{25}\right.$ e $\left.\mathrm{C}_{28}\right)$, aquele que versa sobre a adequação das bibliotecas às necessidades dos estudantes $\left(\mathrm{C}_{25}\right)$ obteve o menor desempenho médio, e aquele que trata do impacto sobre o perfil pesquisador $\left(\mathrm{C}_{6}\right)$ obteve o maior grau de desempenho; e

- Os aspectos considerados mais importantes na visão dos chefes $\left(C_{7}, C_{15}\right.$ e $\left.C_{16}\right)$ também se configuram entre aqueles que apresentaram o maior grau de desempenho.

Com base nos resultados apresentados propuseram-se à coordenação do mestrado em questão as seguintes ações:

- Aumento do acesso bibliográfico e do horário de atendimento da biblioteca do curso;

- Viabilização do acesso remoto ao portal de periódicos da Capes;

- Realização de oficinas e cursos que apoiassem os alunos no acesso ao portal de periódicos da Capes; e

- Desenvolvimento de oficinas sobre práticas de negociação.

Embora a elaboração dos questionários tenha sido feita em uma visão ampla, a coleta de dados no experimento limitou-se à aplicação de questionários em uma única turma de pós-graduação. Em função disso, os resultados do experimento devem ser considerados apenas para esse contexto, não devendo ser feitas extrapolações. Para adotar essa proposta como um sistema de avaliação devem ser incorporados à mesma elementos que permitam uma avaliação do conjunto, incluindo, por exemplo, avaliação dos docentes, bem como a autoavaliação de alunos e docentes.

Como proposta para futuros trabalhos de pesquisas sugere-se a aplicação do questionário junto ao corpo docente, aos coordenadores e aos membros dos comitês de avaliação da Capes - no objetivo de mapear contrastes de percepção entre membros desses diferentes grupos.

\section{Referências}

ALMEIDA FILHO, A. T.; RAMOS, F. S. Avaliação de desempenho de programas de pós-graduação com análise de envoltória de dados. In: ENCONTRO NACIONAL DE ENGENHARIA DE PRODUÇÃO - ENEGEP, 25., 2005, Porto Alegre. Anais... Rio de Janeiro: ABEPRO, 2005. p. 5541-5548.

BARROSO, M. F. C. M. Análise multicritério e avaliação de desempenho docente sob a ótica do corpo discente em instituições de ensino superior. 2002. Dissertação (Mestrado em Engenharia de Produção)-Universidade Estadual do Norte Fluminense, Campos dos Goytacazes, 2002. 
BELLONI, J. A. Uma metodologia de avaliação da eficiência produtiva de universidades federais brasileiras. 2000. 245 f. Tese (Doutorado em Engenharia de Produção e Sistemas)-Universidade Federal de Santa Catarina, Florianópolis, 2000.

BRASIL. Portaria $n^{\circ}$ 080/1998, de 16 de dezembro de 1998. Dispõe sobre o reconhecimento dos mestrados profissionais e dá outras providências. In: NEVES, A. A. B. Presidência da Capes: Coordenação de aperfeiçoamento de pessoal de nível superior. Brasília, 1998. 2 p.

CARVALHO, A. M. A. Monitoramento e avaliação da pós-graduação: algumas reflexões sobre requisitos e critérios. Psicologia USP, v. 12, n. 1, p. 203-221, 2001.

CHARNES, A.; COOPER, W. W.; RHODES, E. Measuring the Efficiency of Decision Making Units. European Journal of Operations Research, v. 2, p. 429-444. 1978. http:// dx.doi.org/10.1016/0377-2217(78)90138-8

COORDENAÇÃO DE APERFEIÇOAMENTO DE PESSOAL DE ENSINO SUPERIOR - CAPES. Regras de avaliação Engenharias 111 (Critérios de avaliação trienal: 2004-2006). Brasília, 2007.16 p. Disponível em: <http://www.capes. gov.br/avaliacao/criterios/avaliacao_trienal_2007.html> Acesso em: out. 2007.

CORDEIRO, C. 0.; PARENTE, R. C. P. A percepção da qualidade dos serviços prestados em uma Instituição de Ensino segundo a ótica do cliente. In: ENCONTRO NACIONAL DE ENGENHARIA DE PRODUÇ̃̃O - ENEGEP, 24., 2004, Florianópolis. Anais... Rio de Janeiro: ABEPRO, 2004.

COSTA, E. L. C. Aplicação de modelo para mapeamento de lacunas de percepção no negócio de e-procurement. 2005. Dissertação (Mestrado em Sistemas de Gestão)Universidade Federal Fluminense, Niterói, 2005.

COSTA, E. L. C.; COSTA, H. G. Modelo para mapeamento de lacunas de percepção no negócio de e-procurement. In: ENCONTRO NACIONAL DE ENGENHARIA DE PRODUÇÃO - ENEGEP, 27., 2007, Foz do lguaçu. Anais... Rio de Janeiro: ABEPRO, 2007.

COSTA, H. G.; FREITAS, A. L. P. Metodologia multicritério para a medição e classificação do grau de satisfação de clientes. In: METROLOGIA 2000 (INTERNATIONAL CONFERENCE ON ADVANCED METROLOGY), 2000, São Paulo. Anais... Brazilian Society of Metrology, 2000.

COSTA, H. G. et al. ELECTRE TRl aplicado a avaliação da satisfação de consumidores. Produção, v. 17, n. 2, p. 230-245, 2007. http://dx.doi.org/10.1590/ S0103-65132007000200002

COSTA, R. C. F.; COSTA, H. G. Aplicação do modelo de "gaps" à avaliação da qualidade em instituição de ensino superior. In: ENCONTRO NACIONAL DE ENGENHARIA DE PRODUÇÃO - ENEGEP, 23., 2003, Ouro Preto. Anais... Rio de Janeiro: ABEPRO, 2003.

FREITAS, A. L. P.; RODRIGUES, S. G.; COSTA, H. G. Emprego de uma abordagem multicritério para classificação do desempenho de instituições de ensino superior. Ensaio: Avaliação e Políticas Públicas em Educação, v. 17, n. 65, p. 655-674, 2009. http://dx.doi.org/10.1590/S010440362009000400006

GRIGOROUDIS, P.; SISKOS, Y. Preference disaggregation for measuring and analyzing customer satisfaction: The MUSA method. European Journal of Operational Research, v. 143, n. 100, p. 148-170, 2001.

KERR-PONTES, L. R. S. et al. Uma reflexão sobre o processo de avaliação das pós-graduações brasileiras com ênfase na área de saúde coletiva. Physis, v. 15, n. 1, p. 83-94, 2005.
LIKERT, R. A. Technique for measurement of attitudes. Archives of Psychology, v. 140, n. 1, p. 5-55, 1932.

LINS, M. P. E.; ALMEIDA, B. F.; BARTHOLO JUNIOR, R. Avaliação de desempenho na pós-graduação utilizando a Análise Envoltória de Dados: o caso da Engenharia de Produção. Revista Brasileira de Pós-Graduação, v. 1, n. 1, p. 41-56, 2004.

MECA, E. F. M. Análise multicritério aplicada à avaliação do sistema de ensino adotado nas Escolas Públicas do Rio de Janeiro. 2006.196 f. Dissertação (Mestrado em Engenharia de Produção)-Universidade Federal Fluminense, Niterói, 2006.

MELLO, J. C. C. B. S.; GOMES, E. G.; LINS, M. P. E. Análise Multicritério da presença da Universidade Federal Fluminense com o uso do Método Macbeth. Produção, v. 11, n. 2, p. 53-67, 2002. http://dx.doi.org/10.1590/ S0103-65132001000200004

MILlER, G. A. The Magical Number Seven, Plus or Minus Two: Some Limits on Our Capacity for Processing Information. Psychological Review, v. 101, n. 2, p. 343-352, 1954. PMid:8022966. http://dx.doi. org/10.1037/0033-295X.101.2.343

MIRANDA, C. M. G.; ALMEIDA, A. T. Avaliação de pós-graduação com Método ELECTRE TRI - 0 caso de Engenharias 111 da CAPES. Produção, v. 13, n. 3, p. 101-112, 2003. http://dx.doi.org/10.1590/S010365132003000300009

MIRANDA, C. M. G.; ALMEIDA, A. T. Visão Multicritério da avaliação de programas de pós-graduação pela CAPES - 0 caso da área Engenharias 111 baseado nos Métodos ELECTRE 11 e MAUT. Gestão \& Produção, v. 11, n. 1, p. 51-64, 2004. http://dx.doi.org/10.1590/ S0104-530X2004000100005

MOREIRA, C. O. F.; HORTALE, V. A.; HARTZ, Z. A. Avaliação da pós-graduação: buscando consenso. Revista Brasileira de Pós-Graduação, v. 1, n. 1, p. 26-40, 2004.

MOTTA, S. S. Avaliação e classificação de professores quanto a produtividade sob a ótica gerencial com o emprego de uma abordagem multicritério. 2002. 180 f. Dissertação (Mestrado em Engenharia de Produção)-Universidade Estadual do Norte Fluminense, Campos dos Goytacazes, 2002.

NEVES, R. B. Integração da análise SWOT com o método electre tri na avaliação do desempenho de programas de pós-graduação. 2005. 203 f. Dissertação (Mestrado em Engenharia de Produção)-Universidade Federal Fluminense, Niterói, 2005.

NEVES, R. B.; COSTA, H. G. Avaliação de programas de pós-graduação: proposta baseada na integração ELECTRE TRI, SWOT e sistema CAPES. Revista Eletrônica Sistemas \& Gestão, v. 1, n. 3, p. 276-298, 2006.

NOGUEIRA, P. Percepção quanto às melhorias viabilizadas pela implantação e prática do gerenciamento da rotina: um estudo de caso em empresa de transporte aéreo. 2004. 124 f. Dissertação (Mestrado Profissional em Sistemas de Gestão)-Universidade Federal Fluminense, Niterói, 2004.

PARASURAMAN, A.; ZEITHAML, V. A.; BERRY, L. L. A conceptual model of service quality and its implications for future research. Journal of Marketing, v. 49, p. 41-50, 1985. http://dx.doi.org/10.2307/1251430

PIQUET, R.; LEAL, J. A. A.; TERRA, D. C. T. Mestrado profissional: proposta polêmica no Sistema Brasileiro de Pós-Graduação - o caso do planejamento regional e 
urbano. Revista Brasileira de Pós-Graduação, v. 2, n. 4, p. 30-37, 2005.

POLITIS, Y.; SISKOS, Y. Multicriteria methodology for the evaluation of a Greek engineering department. European Journal of Operational Research, v. 156, n. 1, p. 223-240, 2004. http://dx.doi.org/10.1016/S03772217(02)00902-5

QUELHAS, 0. L. G.; FARIA FILHO, J. R.; FRANÇA, S. L. B. 0 mestrado profissional no contexto do sistema de pós-graduação brasileiro. Revista Brasileira de Pós-Graduação, v. 2, n. 4, p. 97-104, 2005.

RIBEIRO, L. E. S. Estudo da percepção dos efeitos impactantes do credenciamento de laboratórios de ensaios do INMETRO. 2004. Dissertação (Mestrado Profissional em Sistemas de Gestão)-Universidade Federal Fluminense, Niterói, 2004.

RIBEIRO, T. A. C. Avaliação institucional de IES: um estudo de caso sob a ótica do corpo discente. 125 f. 2003. Dissertação (Mestrado em Engenharia de Produção)-Universidade Estadual do Norte Fluminense, Campos dos Goytacazes, 2003.

RIBEIRO, T. A. C.; COSTA, H. G. Aplicação do método ELECTRE TRI à classificação da percepção do desempenho de IES por parte do corpo discente. In: ENCONTRO NACIONAL DE ENGENHARIA DE PRODUÇÃO - ENEGEP, 25., 2005, Porto Alegre. Anais... Rio de janeiro: ABEPRO, 2005.

ROCHA, N. M. F. Auto-avaliação de centros de pós-graduação: uma proposta em ação. Ensaio: Avaliação e Políticas
Públicas em Educação, v. 14, n. 53, p. 487-506, 2006. http://dx.doi.org/10.1590/S0104-40362006000400006

SANT’ANNA, A. P. Composição probabilística de critérios na avaliação de cursos. Revista Brasileira de Pós-Graduação, v. 2, n. 3, p. 40-54, 2005.

SANT'ANNA, A. P. Modelagem da produtividade e gestão da qualidade acadêmica. Produção, v. 9, p. 5-11, 1999.

SANT'ANNA, A. P. Qualidade, produtividade e gratificação de estímulo à docência. In: SOCIEDADE BRASILEIRA DE PESQUISA OPERACIONAL - SBPO, 33., 2001, Campos do Jordão. Anais... Rio de Janeiro: SOBRAPO, 2001. 14 p.

SANT'ANNA, A. P. Modelagem e avaliação de produtividade de cursos de mestrado em Engenharia de Produção. In: ENCONTRO NACIONAL DE ENGENHARIA DE PRODUÇÃO - ENEGEP, 22., 2002, Curitiba. Anais... Rio de Janeiro: ABEPRO, 2002. 8 p.

SPAGNOLO, F.; SOUZA, V. C. 0 que mudar na avaliação da Capes? Revista Brasileira de Pós-Graduação, v. 1, n. 2, p. 8-34, 2004.

\section{Agradecimentos}

Os autores agradecem à Capes e ao CNPq o apoio financeiro. Os autores também agradecem aos avaliadores e à editoria do periódico as contribuições ao texto.

\begin{abstract}
Professional Master's Programs present major difficulties in their evaluations, since they are the newest modality of stricto sensu graduate program in Brazil. This work presents a methodology to evaluate perceptions on the impact of a Professional Master's Program on the skills of its alumni and on academic aspects of the course implementation. The methodology was applied in a specific situation collecting perceptions from both alumni and their bosses in the organization about the influence of the Master's Program on graduates' skills. Results showed that the respondents have perceived 'self-esteem' and 'researcher's profile' as the skills that were most positively influenced by the program.
\end{abstract}

\title{
Perceptions mapping on the impacts of professional master's programs in the profile of their alumni
} Keywords

Evaluation. Performance. 'Capes'. Professional master’s program. 


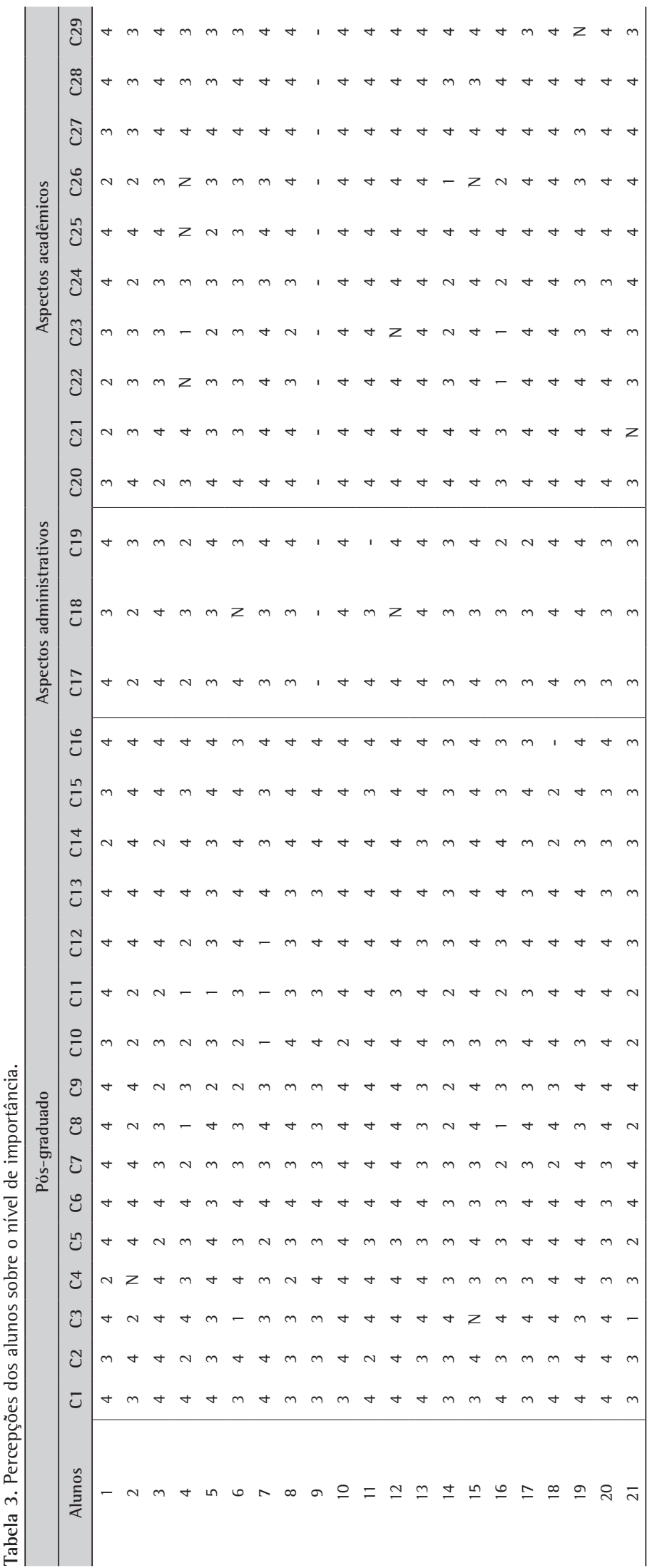


Anexo 2. As Tabelas 4 e 5 apresentam, respectivamente, os resultados dos dados coletados entre os chefes sobre o nível de desempenho e importância dos critérios relacionados na pesquisa.

Tabela 4. Percepções dos chefes sobre o nível de desempenho.

\begin{tabular}{ccccccccccccccccc}
\hline Chefes & C1 & C2 & C3 & C4 & C5 & C6 & C7 & C8 & C9 & C10 & C11 & C12 & C13 & C14 & C15 & C16 \\
\hline 1 & 2 & 2 & 2 & 1 & 2 & 1 & 2 & 2 & 2 & 1 & 2 & 2 & 1 & 2 & 2 & 2 \\
2 & 1 & 1 & 0 & 2 & 2 & 1 & 1 & 2 & 2 & 1 & 1 & 1 & 2 & 1 & 2 & 1 \\
3 & 2 & 1 & 0 & 1 & 1 & 1 & 2 & 1 & 1 & 1 & 1 & 2 & 1 & 1 & 1 & 2 \\
4 & 2 & 2 & 0 & 2 & 2 & 2 & 2 & 2 & 2 & 2 & 2 & 2 & 2 & 2 & 2 & 2 \\
\hline
\end{tabular}

Tabela 5. Percepções dos chefes sobre o nível de importância.

\begin{tabular}{ccccccccccccccccc}
\hline Chefes & C1 & C2 & C3 & C4 & C5 & C6 & C7 & C8 & C9 & C10 & C11 & C12 & C13 & C14 & C15 & C16 \\
\hline 1 & 4 & 3 & 3 & 4 & 3 & 3 & 4 & 4 & 4 & 3 & 3 & 4 & 3 & 3 & 4 & 4 \\
2 & 4 & 3 & 2 & 4 & 4 & 4 & 4 & 4 & 3 & 3 & 3 & 3 & 4 & 3 & 4 & 4 \\
3 & 4 & 3 & 3 & 3 & 4 & 4 & 4 & 3 & 4 & 4 & 4 & 4 & 4 & 4 & 4 & 4 \\
4 & 3 & 3 & 4 & 4 & 4 & 3 & 4 & 4 & 4 & 4 & 4 & 4 & 4 & 4 & 4 & 4 \\
\hline
\end{tabular}

